\title{
Time for the science of pneumonectomyology
}

\author{
Elizabeth A. David, MD, MAS, and Anthony W. Kim, MD
}

\author{
From the Division of Thoracic Surgery, Department of Surgery, Keck School of Medicine, University of Southern \\ California, Los Angeles, Calif. \\ Disclosures: Authors have nothing to disclose with regard to commercial support. \\ Received for publication June 25, 2018; revisions received June 25, 2018; accepted for publication June 27, 2018 \\ Address for reprints: Anthony W. Kim, MD, Division of Thoracic Surgery, Department of Surgery, Keck School \\ of Medicine, University of Southern California Los Angeles, 1510 San Pablo St, Ste 514, Los Angeles, CA \\ 90033 (E-mail: Anthony.Kim@med.usc.edu). \\ J Thorac Cardiovasc Surg 2018;156:1715-6 \\ $0022-5223 / \$ 36.00$ \\ Copyright (C) 2018 Published by Elsevier Inc. on behalf of The American Association for Thoracic Surgery \\ https://doi.org/10.1016/j.jtcvs.2018.06.066
}

The association between right pneumonectomy and mortality is among the long-standing teaching principles in thoracic surgery, with strategies to minimize the risk of bronchopleural fistula being fundamental to mitigating the mortality risk of right pneumonectomy. ${ }^{1}$ Blanc and colleagues $^{2}$ report a comprehensive and quite impressive pneumonectomy series from their institution from 2005 to 2015 that reiterates the association of right pneumonectomy and mortality while offering a slightly new twist to this old problem. The authors describe outcomes for 543 pneumonectomy patients with a focus on 60 patients (11\%) who experienced postpneumonectomy acute respiratory distress syndrome (ARDS) within 30 days of surgery. Operative mortality was highest in patients who experienced ARDS $56.7 \%$ (34 out of 60) and 8.1\% (44 out of 543), overall. Right pneumonectomy and higher Charlson comorbidity index were independent risk factors for ARDS. Independent risk factors of mortality in ARDS patients were severe ARDS, right pneumonectomy, and age with 5-year survival being only $17.6 \%$ for patients with ARDS compared with $43.4 \%$ in patients without ARDS.

The findings of this study echo findings previously reported where low postoperative predictive forced expiratory volume in 1 second and perfusion fraction of the resected lung higher than $35 \%$ were found to be associated with increased risk of ARDS and early mortality in pneumonectomy patients. ${ }^{3}$ That prior investigation found right pneumonectomy to be a risk factor for ARDS on univariate analysis, but the effect did not persist on multivariate analysis. Findings such as these are in keeping with those cited by Blanc and colleagues ${ }^{2}$ in their discussion.

As with all outstanding studies, the authors have provoked us to consider other aspects associated with ARDS and right pneumonectomy. It is interesting to note that not all patients who acquired ARDS in the postoperative setting uniformly had the classic risk factors associated with ARDS, including postoperative pneumonia, severe sepsis, and hemorrhagic shock (presumably necessitating transfusions). As the authors appropriately intimate, this raises level. pneumonectomy.

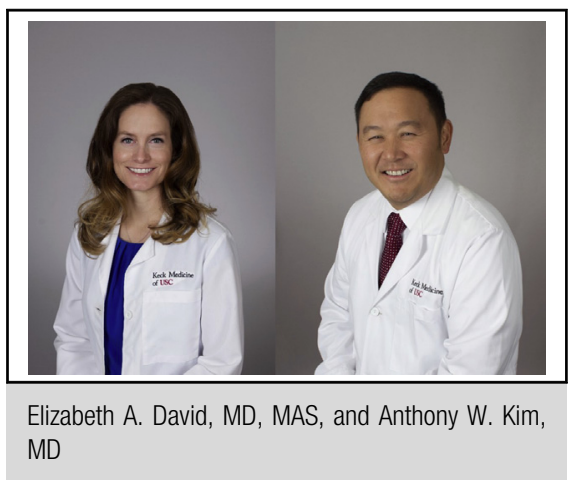

Central Message

An impressive series by Blanc and colleagues highlights the fact that the study of pneumonectomy-related outcomes is an evolving field in need of valuable information such as that described by the authors.

See Article page 1706.

the fact that a significant amount of knowledge regarding the biology and pathophysiology underlying ARDS is founded in a deeper, and yet to be completely elucidated,

The inability of pulmonary function tests to consistently prognosticate ARDS across various studies, including that by Blanc and colleagues, ${ }^{2}$ and the association between the development of ARDS with comorbidities also found in this study, be it determined by specific comorbidities or a score such as the Charlson comorbidity index, suggest that a universally reliable proxy remains absent. Undoubtedly, the authors exercised careful patient selection, including pursuing additional physiologic evaluation of those patients who would require right pneumonectomy. The authors also appropriately emphasize the importance of lung protective strategies as part of both the intraoperative and postoperative management strategies for patients undergoing pneumonectomy. However, even their results demonstrate that despite these best practices, ARDS remains linked strongly to early mortality following right

Clearly there is more work to be done on the part of surgeons and critical care physicians who continue to optimize outcomes for these patients in both the preoperative and perioperative settings, especially for patients who need right pneumonectomy. Because the stakes are so high with right pneumonectomy when ARDS develops, 
considering the incorporation of right heart catheterizations preoperatively may be worthwhile in certain circumstances. Given the association of early mortality with both high perfusion fraction of the resected lung and ARDS, identifying this cohort as at high-risk and directing them toward right heart catheterization may be a reasonable starting point. Obviously, as more information is gathered with respect to pneumonectomy and ARDS, additional, different, and refined criteria may emerge.

Another remarkable finding of this study is the fact that among patients who experienced ARDS, there was a not-so insignificant 5-year survival in this cohort. The authors clearly have shown that the development of ARDS following pneumonectomy is not a universally fatal complication. Whether by design or serendipity (we presume the former) the authors have demonstrated through their excellent clinical expertise that they have evolved knowledge in an area where advancement desperately is needed. We applaud the authors, or dare we say, pneumonectomyologists, for raising the bar and promoting knowledge in what should be considered an area of science that deserves focused study unto itself.

\section{References}

1. Darling GE, Abdurahman A, Yi Q-L, Johnston M, Waddell TK, Pierre A, et al. Risk of a right pneumonectomy: role of bronchopleural fistula. Ann Thorac Surg. 2005;79:433-7.

2. Blanc K, Zaimi R, Dechartres A, Lefebvre A, Janet-Vendroux A, HamilinCanny E, et al. Early acute respiratory distress syndrome after pneumonectomy: presentation, management, and short- and long-term outcomes. J Thorac Cardiovasc Surg. 2018;156:1706-14.e5.

3. Kim JB, Lee SW, Park S-I, Kim YH, Kim DK. Risk factor analysis for postoperative acute respiratory distress syndrome and early mortality after pneumonectomy: the predictive value of preoperative lung perfusion distribution. $J$ Thorac Cardiovasc Surg. 2010;140:26-31. 